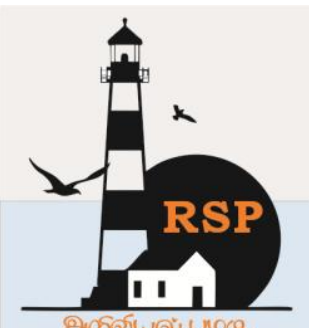

INTERNATIONAL RESEARCH JOURNAL ON ADVANCED SCIENCE HUB

ISSN : $2582-4376$
Open Access

RSP SCIENCE HUB

(The Hub of Research Ideas)

Available online at www.rspsciencehub.com

Special Issue of Second International Conference on Advancements in Research and Development (ICARD 2021)

\title{
Advanced Face Mask Detection System
}

Kalki $N^{1}$, Karthick $M^{2}$, Kavin ${ }^{3}$, Keerthana $S^{4}$, Sangeetha $K^{5}$

${ }^{1,3,4} U G$ Scholar, Department of Computer Science and Engineering, SNS College of Technology, Coimbatore, India.

${ }^{2,5}$ Assistant Professor, Department of Computer Science and Engineering, SNS College of Technology, Coimbatore, India.

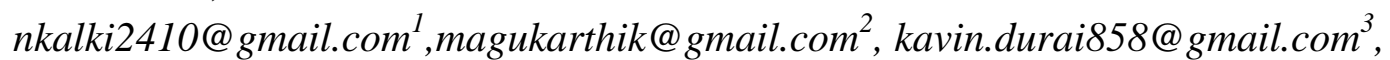
keerthanassm04@gmail.com ${ }^{4}$,sangeethprakash@gmail.com ${ }^{5}$.

\begin{abstract}
Pandemic COVID-19 novelly induced corona viruses that are continually spreading all over the world. In most areas of COVID-19 affect creation fallen. There is a crisis in the health sector. There have been several precautionary steps brought to minimize the propagation of this disease, with a mask. We are proposing a system that reduces COVID-19 development, identifying individuals who don't use a facial mask in a smart city system that monitors all public places with CCTV cameras. The appropriate authority is alerted by the city network whilst an individual is identified without a mask. Architecture for a data set that includes images of individuals with and without masks from various sources can train machine learning. A qualified architecture has achieved precision in identifying individuals from previously unknown testing data with and without a face mask
\end{abstract}

Keywords: Mask detection, CCTV, Machine learning

\section{Introduction}

Formed by COVID-19 the pandemic disease that has a powerful impact on humankind and thus our way of seeing our environment and everyday life has created the most important international health concern in the world. In the city centre of China, in December 1961, when 7711 people died, 170 people died before a global coronavirus was announced and the World Health Association designated the world as COVID-19, a brand new severe infection of the respiratory tract occurred (coronavirus sickness 2019). Phase of the World COVID-19, infected more than $13,039,853$ people and reported 581,659 deaths in more than 200 different countries, has been registered in the Health Organization (WHO). Compared with just 1 respiratory disease chronicles. However, as we all know the transmission of the new coronavirus caused by corona virus (COVID 19) can also be a good carrier with no Covid indications, we all know. Uniquely, it was the result of human-tohuman transmission. No study on antiviral medications or vaccines that are clinically approved to assess the effectiveness of COVID-19 is available to date. It has rapidly unfolded across the world, bringing the entire human population great challenges to Economic, environmental, social and health problems. The UN agency immediately recommends that \{persons \} face the most vulnerable masks in order to prevent virus transmission and jointly recommends maintaining a social gap of at least $2 \mathrm{~m}$ between people in order to stop unpopulation. Moreover, a number of public service providers require clients to use the service, provided they wear masks and observe social distance safely. Face mask identification and 
secure social distance monitoring have become an extremely important activity for the global community of laptop vision. This paper outlines approaches to avoid the disappearance of the virus by real time as people watch for safe social isolation and wear public face masks.[1-5]

\subsection{Face Recognition}

Face recognition is conducted with several algorithms based mainly on or model. Most feature-based algorithms are used in real time in security systems. The main elements of Analysis and Linear Discriminant Analysis (LDA) are ar cost-effective in the extraction of recognition options. However Linear Discriminant Analyses (LDA, by its Spanish acronym), once giant coaching sets are recognized in their identity, outperforms primary element analysis (PCA) algorithm. In addition, LDA discriminates much of the info gift in the picture by computing intragroup scatter matrices and resting category. Miss handling information containing normalized face images, popularity is done via the LDA rule in the vehicle safety system. In the information called coaching images and the camera non-inherited face picture, which is called the check image, the LDA extracts the function of the keep images. The check image must then be compared to the information pictures and the image classifier used in the rule determines if the geometer distance and the edge value are the best known or unknown mistreatment. The distance between the geometers is determined between the corresponding weights and the picture that generates the minimum distance is better balanced with the picture. When a mathematician has less than the brink value, and the individual is classified as unknown or unauthorized when the distance values are above the threshold, they are categorized as proverbial or allowed.[6-10]

\section{Problem Identification 2.1 Existing Method:}

The project uses profound techniques for the distinguishing biometric authentication and recognizes whether or not the individual is wearing a mask. The collected data set includes $224 \times 224$ component resolution for 205000 images and achieved a related grade accuracy ratio of ninetysix on the output of the trained model. The device develops a Raspberry Pi-based time facemask that recognizes and captures the facial picture if the detected person has not an exterior.

\subsection{Proposed System}

The framework proposed helps ensure the safety of people in public places by automatically securing them in a secure social distance or not and collectively by detecting whether or not they wear an individual mask. The system used a CNN identity verification system based on the learning machine to see whether or not the individual is sporting mask. When the device detects if an individual is not sports mask, it captures the picture and sends the information to the city authority in the cloud and collectively alerts the system to mask voice.

\section{Methodology}

The Network Video Recorder (NVR) camera provides the stream RTSP pattern and converts these frameworks into grey to improve speed and accuracy, and sends the system into the model for any raspberry pi4 process. Since we used the MobileNetV2 core detective model, MobileNetV2 offers an excessive price advantage in comparison to the second CNN standard model. The SSD MultiBox Detector also contains a neural specification of over-size for associated images such as ImageNet and Passcal VOC already eligible for high-level image grades in nursing. With ImageNet weight, we are inclined to load the Mobile Net V2.leave the network behind and build a new, sweeping FC head, link it with very cheap instead of the new head, freezing very low network layers. During fine standardization, the weights of these basic layers cannot be changed, while the maximum weight of the layers can be modified. The model is then collected and trained when the information is prepared and then the model style is up for fine standardization. When the look is ready very little learning speed is used to assure that already learned convolutionary filters do not significantly differ and experiments with OpenCV, Tensor Flow Deep Learning patterns and pc There is no vision so that we can examine in the number of time video streams a stable Social divide between people and masks. The key contribution of the planned system is three parts: the human identification, the safe distance operation between the identified persons and the detection of masks. 91 per cent of mAP is supported by the MobileNet 


\section{www.rspsciencehub.com}

V2 and OpenCV single-shot object (SSD) pattern, which outperform the comparable R-CNN, which is more advanced. Anyone who has been detected shows a bounding box. Although in aterribly frame SSD can research multiple artefacts, this technique only detects one person. In calculating the differences between two people, the personal divide from the camera is determined using the pattern triangles of similitude technique, in which the distance from the camera is calculated, tending to the actual height $\mathrm{H}=165 \mathrm{cms}$.pattern these values The camera gap is further determined in the following formulation pattern: $\mathrm{F}=(\mathrm{D} \times \mathrm{P})$ Then we prefer to use the height of $\$ 64,000 \mathrm{H}$, a halfheight $\mathrm{P}$ and thus the distance $\mathrm{F}$ of the A camera live the camera's distance from the person. Additionally, the camera gap is calculated by the following pattern: $(\mathrm{H} \times \mathrm{F}) / \mathrm{P} . \mathrm{D} 1=\mathrm{We}$ are tender to measure the distance between 2 people at intervals of the video once we have shredded the person's depth at intervals on the frame.

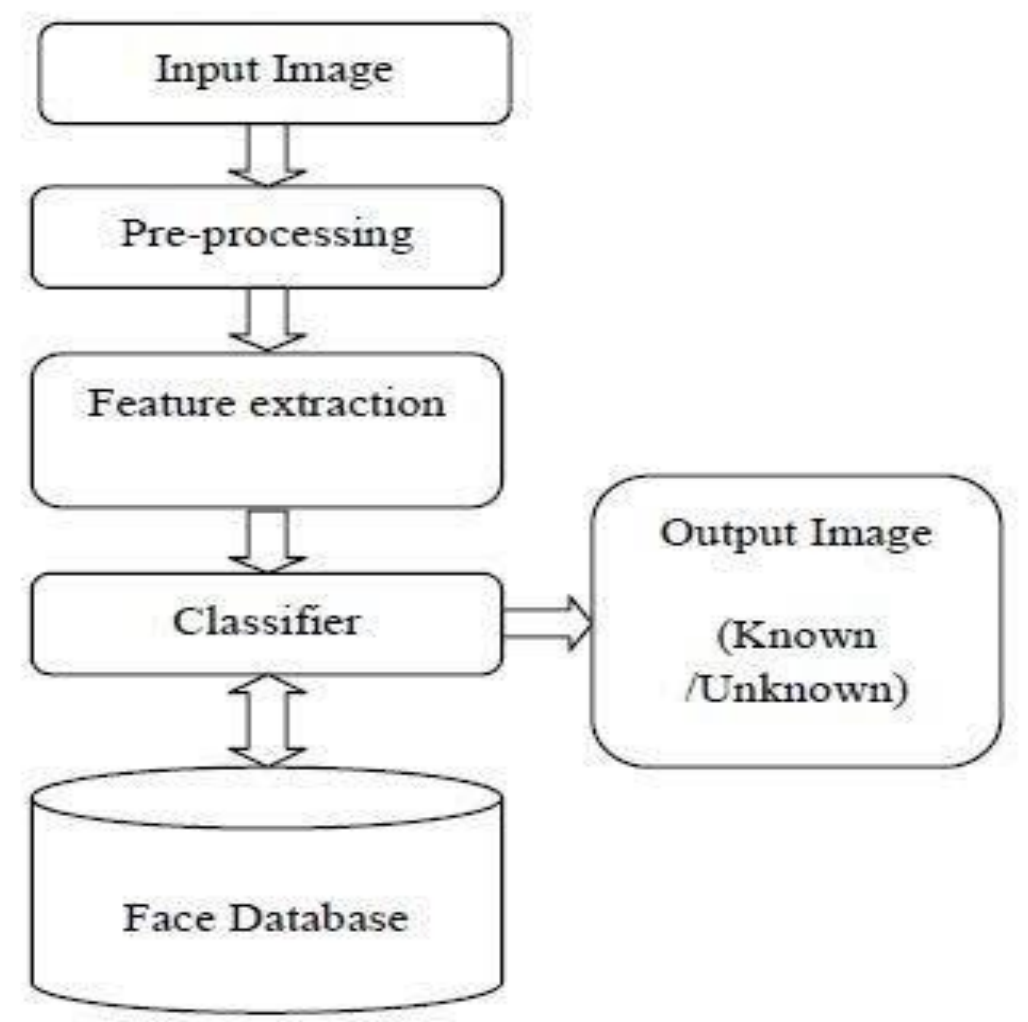

\section{Output Design}

Quality output that satisfies the highest demands of consumers and shows the data clearly. Users and completely different system outputs are communicated in any system outcomes of the operation. In output mode this is decided, but information can be transferred for immediate use and the output of the text can be booted. The most important and direct provision of user data is. Economic and intelligent success improves the system's relationship and lets users determine. One or more of the following goals must be accomplished across the device's output spectrum. Transmit data on past events, current position or future predictions. Signs key incidents, possibilities, issues or alerts. Trigger behavior and perform any action related to it Definition of module: Pre-processing: we prefer to make a bar chart try to avoid alterations of light after we have cut off the region of your face. A conical mask is often used to eliminate the large array of corner regions and the collar in order to reduce the unhanding effects in those areas. However, for outof-session images the masking phase is not used, as the photos are of little quality and scale. Deletion feature: Our facial description will be used once the pre-processing stage by the native binary pattern (LBP) operator. LBP is among the most successful descriptors of acting texture and is widely used in various uses. It has been checked to be rather discriminatory and to its main blessings: its variability in grey monotonous changes and the effectiveness of the procedure build it acceptable for tight image analysis tasks. Compare a sample 


\section{www.rspsciencehub.com}

gallery and then supply accurate data to make a right judgment and check a valid user's validity or incorrect user maltreatment for front pictures taken by an embedded VEHICLE camera. Verification system: Verification system: Object Detection: This module is used to find the wear mask of the user and to mistreat OpenCV integral hair cascade profile detector with any object (strike, shot, knife). Object detection. The best rotation angle for increasing face detection is used by the user with Brobdingnagian mask in the event of violence.

\section{Conclusion:}

This project is focused on the recognition of facemasks through Convolution Neural Networks in real time. For facial mask detection, this process produces accurate and fast results. The test results indicate that people wear a facemask and not wear a facemask was identified with distinct precision. There have therefore been several precautionary steps to combat coronavirus. Among them, the main thing, is washing hands, keeping safe distance, wearing a mask, not touching the eyes, nose and mouth. COVID-19 is a human-to-human disease that can be controlated by the proper use of a face mask. If people maintain strict social distance and use facial masks, the propagation of COVID-19 can be restricted. Unfortunately, the laws that accelerate the spread of this virus are not being obeyed correctly by people. It will help to reduce the distribution of coronaviruse by detecting people who do not comply with the regulations and informing the corresponding authorities.

\section{References:}

[1].KresimirDelac and MislavGrgic,"Face recognition", I-TECH Education and Publishing, Vienna,Austria,2007

[2].WeilongChen, MengJooEr, "Illumination Compensation and Normalization for Robust FaceRecognition Using Discrete Cosine Transform in Logarithm Domain," IEEE Transactions On Systems, Man And Cybernetics, Vol.36,No.2, April 2006

[3].Jie Wang, K.N. Plataniotis, A.N. Venetsanopoulos, "Selecting discriminant eigenfaces for face recognition", Pattern Recognition Letters 26 (2005), science direct

[4].M.Turk. and A.Pentland., "Face recognition using eigenfaces", Proceedings of IEEE
Volume 03 Issue 03S March 2021

Conference on Computer Vision and Pattern Recognition, Maui, Hawaii, pp. 586-591, 3-6 June 1991.

[5].M. Turk, A. Pentland, "Eigenfaces for Recognition", Journal of Cognitive Neurosicence, Vol. 3, No. 1, pp. 71-86, 1991

[6].Dr. Arti Khaparde, Sowmya Reddy.Y Swetha Ravipudi ,'Face Detection Using Color Based Segmentation and Morphological Processing A Case Study," Whitepaper

[7].Access Control, Technology — secuwatch, Whitepaper, September 27, 2007

[8].Smrithy.K.M ,D.PrasannaVadana, Sarath S Nair,' Image Processing Based Attendance Marking System'

[9].P Bagavathy, $\mathrm{R}$ Dhaya and $\mathrm{T}$ Devakumar (2011), Real time car theft decline system using ARM processor, Proceedings of International Conference on Advances in Recent Technologies in Communication and Computing, pp. 101- 105.

[10].S K Hese and M R Banwaskar (2013), Performance Evaluation of PCA and LDA for Face Recognition, International Journal of Advanced Research in Electronics and Communication Engineering, Maharashtra, India, 2, 2, pp.149-152. 